\title{
Midlife moderation-quantified healthy diet and 40-year mortality risk from CHD: the prospective National Heart, Lung, and Blood Institute Twin Study
}

\author{
Jun Dai ${ }^{1,2 *}$, Ruth E. Krasnow ${ }^{3}$ and Terry Reed ${ }^{4}$ \\ ${ }^{1}$ Master of Public Health Program, Department of Public Health, Des Moines University, Des Moines, IA 50312, USA \\ ${ }^{2}$ Division of Epidemiology, Vanderbilt University Medical Center, Nashville, TN 37203, USA \\ ${ }^{3}$ Center for Health Sciences, Biosciences Division, SRI International, Menlo Park, CA 94025, USA \\ ${ }^{4}$ Department of Medical and Molecular Genetics, Indiana University School of Medicine, Indianapolis, IN 46202, USA \\ (Submitted 23 October 2015 - Final revision received 26 February 2016 - Accepted 21 March 2016 - First published online 18 May 2016)
}

\section{Abstract}

It is unknown whether influences of midlife whole diet on the long-term CHD mortality risk are independent of genetic and common environmental factors or familial predisposition. We addressed this question prospectively using data from the National Heart, Lung, and Blood Institute Twin Study. We included 910 male twins who were middle-aged and had usual diet assessed with nutritionist-administered, crosschecked dietary history interview at baseline (1969-1973). Moderation-quantified healthy diet (MQHD), a dietary pattern, was created to evaluate a whole diet. Primary outcome was time-to-CHD death. Hazard ratios (HR) were estimated using frailty survival model. Known CHD risk factors were controlled. During the follow-up of 40 years through 31 December 2009, 113 CHD deaths, 198 total cardiovascular deaths and 610 all-cause deaths occurred. In the entire cohort, the multivariable-adjusted HR for the overall association (equivalent to a general population association) was $0.76(95 \%$ CI $0 \cdot 66,0 \cdot 88)$ per 10-unit increment in the MQHD score for CHD, and the multivariable-adjusted HR for a twin with a MQHD score ten units higher than his co-twin brother was 0.79 (95\% CI 0.64, 0.96, $P=0.02)$ for CHD independent of familial predisposition. Similar results were found for a slightly more food-specified alternative moderation-quantified healthy diet (aMQHD). The between-pair association (reflecting familial influence) was significant for CHD for both MQHD and aMQHD. It is concluded that associations of MQHD and aMQHD with a lower long-term CHD mortality risk are both nutritionally and familially affected, supporting their use for dietary planning to prevent CHD mortality.

\section{Key words: CHD: Mortality: Diets: Twin studies}

CHD remains the leading cause of death in the USA ${ }^{(1)}$, and dietary intake is an important attributable factor. Secular changes in cardiovascular and CHD death ${ }^{(2)}$ and food consumption ${ }^{(3)}$ strongly suggest nation-wide common environmental rather than genetic influences on these phenotypes. Twin studies have demonstrated the genetic impact on $\mathrm{CHD}^{(4,5)}$, and dietary intake $^{(6)}$, as well as influences of familial environment ${ }^{(4,7)}$ and shared non-familial environment ${ }^{(4,8,9)}$ on $\operatorname{diet}^{(7-9)}$ and $\mathrm{CHD}^{(4,10)}$. To date, there is no evidence from a 40-year dietary randomised controlled trial targeting CHD. Therefore, it remains unclear whether diet related to very long-term (like 40 years in the reported study) CHD mortality risk is genetically, commonenvironmentally or familially independent.

The nature of diet is complex. Humans eat a variety of foods containing numerous dietary compounds. Food items can be processed or cooked in diverse ways. A combination of all consumed foods and beverages with the synergy ${ }^{(11)}$ and the antagonism among them when interacting with human bodies, defined as a whole diet, affect health and disease. Whole diet can be evaluated through dietary pattern or diet quality in a population study, as reviewed previously ${ }^{(12)}$. There are two limitations in previously traditional observational studies of whole diet ${ }^{(13-16)}$ : potential confounding from genetic and environmental influences was not well controlled, and moderate consumption was not considered in the evaluation of whole diet in relation to long-term CHD.

To overcome these two limitations, we created the plantfood-dominant dietary pattern ${ }^{(13-20)}$ with an attempt to incorporate the well-known nutritional concept of moderate consumption into the quantification of a whole diet ${ }^{(21)}$. Next, using a co-twin control design, we aimed to address whether this dietary pattern was prospectively related to long-term CHD mortality risk, independent of genetic factors and common environment, or familial predisposition, and we tested the contribution from genetic and environmental factors to the association. Our outcome was time-to-event, where the primary event was CHD deaths and the secondary event was deaths from total CVD and all causes.

Abbreviations: aMQHD, alternative modified Mediterranean-style diet; DZ, dizygotic; MQHD, modified Mediterranean-style diet; HR, hazard ratio; MZ, monozygotic.

* Corresponding author: Dr J. Dai, email jun.dai@dmu.edu 


\section{Methods}

\section{Study population}

As widely described ${ }^{(22-24)}$, initiated in 1969 , the prospective National Heart, Lung, and Blood Institute (NHLBI) Twin Study enrolled 514 middle-aged, white male veteran twin pairs (254 monozygotic (MZ) and 260 dizygotic (DZ) twin pairs ${ }^{(22)}$ ) who were born between 1917 and 1927 and aged 42-55 years at the baseline examination (1969-1973). All twins were physically examined at baseline and during follow-up investigations. Zygosity was ascertained by eight RBC antigen groups and later variable number of tandem repeat DNA markers ${ }^{(25)}$. The Institutional Review Board at each examination site approved the study protocol, and all twins gave written informed consent. Exclusion criteria a priori were as follows: (1) no dietary data available, and (2) daily total energy intake $<2092 \mathrm{~kJ}(500 \mathrm{kcal})$ or $>25104 \mathrm{~kJ}(6000 \mathrm{kcal})^{(26)}$. Baseline dietary data were not collected among fifty-nine twin pairs. No twins had implausible total energy intake. A total of 910 twins (234 MZ and $221 \mathrm{DZ}$ pairs) were included in the analyses with cumulative 26672 person-years during the 40-year follow-up period.

\section{Baseline diet assessment}

Usual dietary data were obtained through a standardised nutritionist-administered, cross-checked, dietary history interview $^{(27)}$ validated in the Framingham Study ${ }^{(28,29)}$. Daily food intake amount was calculated from food intake frequency and the serving size. Daily energy intake and daily nutrient intake in gram weight were calculated.

\section{Moderation-quantified healthy diet score}

With an attempt to incorporate the well-known nutritional concept of moderate consumption, we used a scoring strategy modified from Rumawas et $a l^{(21)}$. The moderation-quantified healthy diet (MQHD) score contained fourteen components (Table 1): total grains, fruits, vegetables, dairy products, alcohol $^{(30)}$, fish, poultry, red meats, nuts and legumes, potatoes, eggs, sweets, ratio of fried meat:non-fried meat and ratio of unsaturated fatty acids:SFA ${ }^{(31)}$. The amount of a food item or group was measured in servings, and was adjusted for $10460 \mathrm{~kJ} / \mathrm{d}(2500 \mathrm{kcal} / \mathrm{d})$. Thus, the individualised food consumption amount could be calculated as the number of servings of a food group for the reference value for maximal component score (Table 1$) \times$ individualised total energy requirement in $\mathrm{kJ}$ (kcal)/(10460 kJ or $2500 \mathrm{kcal})$ '. A value for each component was calculated with a penalty for excessive intake $^{(21)}$, except for the ratios. If the amount of each component was below the reference, a value ranging from 0 to 10 was calculated as ' $10 \times$ the consumption amount divided by the reference'. If the consumption amount of each component was greater than the reference, a value was given as ' $10-(10 \times$ (the consumption amount-the reference)/the reference)'. If the value was negative, it was converted into 0 . A value of 0 was assigned for a ratio of fried meat:non-fried meat $>0 \cdot 8$. If the ratio of fried meat:non-fried meat was $\leq 0 \cdot 8$, a value was calculated as
' $10-10 \times$ the ratio divided by the reference'. For ratio of unsaturated fatty acids:SFA, if the ratio was $\leq 4$, a value from 0 to 10 was calculated as " $10 \times$ the ratio divided by 4 (31) ; if the ratio was $>4$, a value of 0 was assigned because of the concern for oxidative stress. A total score was generated through summing the value from each of fourteen components.

We constructed a slightly more food-specified version: alternative moderation-quantified healthy diet (aMQHD). The aMQHD score included four additional components: the presence of lamb or veal consumption, the presence of skimmed milk consumption, ratio of white meat:red meat and ratio of ice cream:dairy product consumption. Addition of these four components that were relevant to what Americans consumed might help dietary or eating planning to a certain degree, as such planning often needs detailed food specification. A value of 10 was assigned if lamb or veal was consumed, skimmed milk was consumed, if the ratio of white meat:red meat was $\geq 1$ or if the ratio of ice cream consumption:total dairy product consumption was $\leq 0 \cdot 1$. Otherwise, a value of 0 was given. A total score was the sum of values from each of eighteen components. The aMQHD score was the standardised total score with a theoretical range from 0 to 100 . Because of the enhanced specification, it was less generalisable than MQHD.

Both MQHD and aMQHD scores were standardised to a 0 to 100 scale as ' $100 \times$ total score divided by the theoretical maximum score of 140 for MQHD and 180 for aMQHD' with a theoretical range from 0 to 100 . A higher value indicates greater conformity to the healthy whole diet. Because of the penalty for excessive intake and the assignment of a low value for the intake below the recommended consumption amount $^{(21)}$, we translated the nutritional concept of 'moderate consumption' to our scoring algorithm.

\section{Other baseline variables}

As described previously ${ }^{(22)}$, through in-person interview and physical examination, data were recorded for demographic, socio-economic, lifestyle, familial, anthropometric, biochemical and clinical factors. Information on current use of medications was collected. Heart disease and other CVD were diagnosed by the physician at baseline ${ }^{(32)}$

\section{Assessment of end points and follow-up}

Vital status and the cause and date of death were ascertained through medical records in four active follow-up examinations and later on using death certificates or the National Death Index through 31 December $2009^{(32)}$. As previously described ${ }^{(22,32)}$, physicians assigned corresponding International Classification of Diseases, Ninth Revision codes for morbidity outcomes. Death certificates or the National Death Index coded to the ninth revision codes were obtained for decedents. The primary end point was death from CHD (410-414). Secondary end points were death from all CVD (390-398, 402, 404, 410-438) and all causes. Subjects were considered lost to follow-up if a death certificate or coding from the National Death Index could not be traced. The lost-to-follow-up twins were included in this study and treated as if they were alive at the date of the 
end of the study ${ }^{(22)}$. The follow-up was terminated at the date of death, end of follow-up or loss to follow-up, whichever occurred first.

\section{Statistical analysis}

To avoid model overfitting, particularly for analyses among MZ twins, we constructed a modified Framingham Risk Score ${ }^{(22,33)}$. This risk score consisted of seven cardiovascular risk factors (age, smoking, systolic and diastolic blood pressure, cholesterol in HDL and LDL and diabetic status ${ }^{(33)}$ ), and was associated with coronary heart mortality risk in our cohort (hazard ratio (HR) per unit increment in the risk score, 1.08; 95\% CI, 1.05, 1.11). Intraclass correlation coefficient for the diet score was estimated with a random coefficient model. Heritability was calculated using Falconer's method ${ }^{(34)}$. Factors shared between co-twins could be categorised into shared genetic and common environmental factors between co-twins, or into familial factors (a combination of genes and familial environment) and environment shared outside of family (i.e. shared non-familial environment, such as local food supply/accessibility/processing/service/retail settings, recreational facilities, school and workplaces $^{(22)}$ ) (Fig. 1).

We used a frailty survival model to estimate $\mathrm{HR}^{(22,35)}$ to account for clustering within a twin pair ${ }^{(22)}$. In all models, the outcome was time-to-event. In the overall association analysis, we treated the study population as a general population through accounting for clustering. The exposure variable was a diet score and was used as a continuous variable after we confirmed the linearity using restricted cubic splines ${ }^{(36,37)}$. In analyses of the association between the diet score and the outcome controlling for familial predisposition and shared non-familial environment (a matched analysis for the co-twin control design), we used a within-pair and between-pair effect model $^{(38)}$. The within-pair effect variable was the exposure. The between-pair effect variable was both a predictor and a matching factor representing a combination of factors shared between co-twins (Fig. 1). The within-pair effect of diet score was calculated as the deviation of a twin's diet score from the mean diet score of his twin pair, and controls for confounding from shared factors (Fig. 1) ${ }^{(38)}$. If the interaction was not statistically significant, we used data pooled by zygosity. If otherwise, zygosity-specific analyses would be considered.

Other individual-level covariates/predictors were controlled in the model. We controlled for total energy intake (continuous) in the basic model. Then, we additionally adjusted for known CHD risk factors, including socio-economic factors (years of education (continuous) ${ }^{(39)}$ ), lifestyle factors (marital status (never, not married currently and married currently), BMI (continuous)), modified Framingham Risk Score (continuous) and use of antihypertensives (yes/no). Multiple imputations were used to account for missing data and to obtain fully adjusted parameter estimates.

We performed a confirmatory analysis, as a single dietary measure could attenuate the association because of the misclassification. Given the comparable classification between a multi-measure and a single measure for those at extreme intakes $^{(40)}$, we compared those in the top score quartile with those in the bottom one in relation to coronary heart death risk. Sensitivity analyses were performed using several slightly modified versions of diet scores, and after exclusion of twins with baseline diabetes and CHD. We also performed secondary analyses using the Mediterranean diet score constructed according to the algorithm published by Trichopoulou et $a l^{(13)}$.

All analyses were conducted with SAS software version 9.2 (SAS Institute). Significance levels were set at 0.05 (two-sided).

\section{Results}

\section{Components of whole diet}

Table 1 shows each score component, food items consisting of each component, the reference value for the maximal score of 10 for each component and the median value of each component in the studied cohort.

\section{Characteristics of the study participants}

A total of 910 twins (455 twin pairs) were followed up to 40 years with a median of $32 \cdot 0$ (interquartile range $23 \cdot 3-37 \cdot 3$ ) years. There were 113 CHD deaths, 198 cardiovascular deaths and 610 all-cause deaths. The mean age at baseline was 48.0 (range 42-55) years. MQHD score ranged from 10 to $57 \cdot 1$. Men with a higher diet score had a lower energy intake, higher percentages of energy content from both protein and carbohydrates, a lower percentage of energy content from dietary total fat, SFA and MUFA, more years of education and higher systolic blood pressure (Table 2). In all, nineteen twins out of the 910 twins were lost to follow-up. The lost-to-follow-up twins were similar to other twins, except that they were less likely to be currently married (data not shown).

\section{Overall associations (i.e. general population associations)}

Table 3 shows that a greater MQHD score, meaning a dietary pattern that would be considered related to better health than a lower score, was statistically significantly associated with a lower mortality risk from specific and all causes after energy intake adjustment in the whole cohort (i.e. MZ and DZ combined). Multivariable adjustment did not materially change the association for specific and all causes: HR for each 10-unit increment in MQHD score was 0.76 (95\% CI $0.66,0.88$ ) for CHD mortality risk, 0.87 (95\% CI 0.79, 0.96) for cardiovascular mortality risk and 0.95 (95\% CI 0.91, 0.996) for all causes (Table 3).

The heritability was $21 \%$ for MQHD score and $4.5 \%$ for aMQHD (online Supplementary Table S1). Variation in factors shared between co-twins (i.e. shared genetic and common environmental factors including familial predisposition factors and shared non-familial environment in Fig. 1) explained $24 \%$ of variation in MQHD score and $27 \%$ in aMQHD score (online Supplementary Table S1). These shared factors that made co-twins similar were next controlled in within-pair analyses. 
Table 1. The reference value for the maximal score of 10 for each component of moderation-quantified healthy diet score and alternative moderation-quantified healthy diet (MQHD) score among 910 twins*

(Medians and interquartile ranges (IQR))

\begin{tabular}{|c|c|c|c|c|c|}
\hline \multirow[b]{2}{*}{ Food groups } & \multirow[b]{2}{*}{ Food items } & \multirow{2}{*}{\multicolumn{2}{|c|}{ Reference value for the maximal score of 10}} & \multicolumn{2}{|c|}{ Intake } \\
\hline & & & & Median & IQR \\
\hline \multicolumn{6}{|c|}{ Individual score components common to both MQHD score and aMQHD score } \\
\hline & & Servings/d & & Servings/d & \\
\hline Grains & Spaghetti/pasta, rice, cereal, bread, sweet roll/donut, cake/pie/pastry & 8 & & 6 & $4-7$ \\
\hline Fruits & Fruits, fruit juice & 3 & & 2 & $1-3$ \\
\hline Vegetables & Salad, cooked vegetables & 6 & & 2.5 & $2-3$ \\
\hline Dairy products & $\dagger \ddagger$ & 2 & & $4.5 \dagger$ or $2.0 \ddagger$ & $3.1-6.0 \dagger$ or $1.2-3.0 \ddagger$ \\
\hline Alcohol & Beer, wine, other alcoholic beverages (spirit, cocktail) & $\stackrel{2}{2}$ & Servings/d & $\begin{array}{l}0.6 \\
\text { Servings/week }\end{array}$ & $0-1.6$ \\
\hline Fish and shellfish & Shellfish, non-shellfish & 4 & 0.57 & 1.5 & $0-3$ \\
\hline Poultry & Chicken/turkey, fried chicken & 2 & 0.29 & 2 & $1-3$ \\
\hline Red meat & $\begin{array}{l}\text { Pork, ham, bacon, sausage, steak, pot roast, beef, hamburger, } \\
\text { luncheon meat, hotdog, liver, sweetbreads, brain, fried meat }\end{array}$ & 3 & 0.43 & 14 & $11-18$ \\
\hline Eggs & Eggs, fried egg & 3 & 0.43 & 6 & $3-11$ \\
\hline Nuts & Peanut butter, nuts & 4 & 0.57 & 3 & $1-5$ \\
\hline Potatoes and maize chips & Potatoes, fried potatoes, potato chips, maize chips & 3 & 0.43 & 9 & $5-12$ \\
\hline Food groups & Food items & 3 & 0.43 & 19 & $12-17$ \\
\hline Ratio of unsaturated fatty acids:SFA & - & 4 & 0.57 & 1.56 & $1.39-1.74$ \\
\hline Ratio of fried foods:non-fried foods§ & - & 0.8 & & 0.35 & $0.18-0.60$ \\
\hline \multicolumn{6}{|c|}{ Individual score components unique to aMQHD score } \\
\hline Lamb or veal & Lamb or veal & $>0$ & & $0 \|$ & $0-0$ \\
\hline Skimmed milk & Skimmed milk & $>0$ & & 0थ & $0-0.29$ \\
\hline Ratio of white meat:red meat ${ }^{\star *}$ & - & $\geq 1$ & & 0.25 & $0.12-0.40$ \\
\hline Ratio of ice cream:dairy products & - & $\leq 0.1$ & & 0.04 & $0-0.04$ \\
\hline
\end{tabular}

aMQHD, alternative modified Mediterranean-style diet.

Servings were based on daily $10460 \mathrm{~kJ}(2500 \mathrm{kcal})$.

† Dairy products referred to all milk and milk products including whole milk, skimmed milk, chocolate milk, ice cream, milk-containing products such as pudding, butter and cheese.

₹ Dairy products the same as defined above, with exclusion of butter.

$\S$ Fried food included fried potatoes, potato chips, fried eggs, fried meat, fried chicken, fried fish and fried shellfish

\| Servings/week.

II Servings/d.

${ }^{* *}$ White meat includes seafood, fish and poultry. 
Table 2. Baseline characteristics of twins in relation to a continuous moderation-quantified healthy diet (MQHD) score in the whole cohort pooled by zygosity*

(Numbers and percentages; mean values with their standard errors

\begin{tabular}{|c|c|c|c|c|}
\hline \multirow[b]{2}{*}{ Variables } & \multicolumn{2}{|c|}{ Whole cohort $(n 910)$} & \multicolumn{2}{|c|}{$\begin{array}{l}\text { Coefficients of regression of predictors on } \\
\text { diet score }\end{array}$} \\
\hline & $\%$ & $n$ & MQHD score & $P$ \\
\hline \multicolumn{5}{|l|}{ Total energy content $(\mathrm{kJ} / \mathrm{d})$} \\
\hline Mean & \multicolumn{2}{|c|}{8464} & & \\
\hline SE & \multicolumn{2}{|c|}{100} & & \\
\hline Total energy content (kcal/d) & & & -0.003 & $<0.001$ \\
\hline $\begin{array}{l}\text { Mean } \\
\text { SE }\end{array}$ & 2023 & 24 & & \\
\hline Percentage of energy content from total fat (\%) & & & -0.38 & $<0.001$ \\
\hline $\begin{array}{l}\text { Mean } \\
\text { SE }\end{array}$ & \multicolumn{2}{|c|}{$40 \cdot 2$} & & \\
\hline Percentage of energy content from carbohydrates (\%) & & & 0.25 & $<0.001$ \\
\hline Mean & \multicolumn{2}{|c|}{44.7} & & \\
\hline SE & \multicolumn{2}{|c|}{0.3} & & \\
\hline Percentage of energy content from protein (\%) & & & 0.31 & $<0.001$ \\
\hline Mean & \multicolumn{2}{|c|}{$15 \cdot 2$} & & \\
\hline SE & \multicolumn{2}{|c|}{0.1} & & \\
\hline Percentage of energy content from SFA (\%) & & & -0.77 & $<0.001$ \\
\hline Mean & \multicolumn{2}{|c|}{$15 \cdot 7$} & & \\
\hline SE & \multicolumn{2}{|c|}{0.1} & & \\
\hline Percentage of energy content from MUFA (\%) & & & -0.63 & $<0.001$ \\
\hline Mean & \multicolumn{2}{|c|}{$20 \cdot 9$} & & \\
\hline SE & & & & \\
\hline Percentage of energy content from PUFA (\%) & & & 0.08 & 0.70 \\
\hline Mean & & & & \\
\hline SE & & & & \\
\hline Age (years) & & & -0.06 & 0.52 \\
\hline Mean & & & & \\
\hline SE & & & & \\
\hline Smoking & & & & \\
\hline Never smokers & 42 & 383 & Ref. & \\
\hline Former smokers & 10 & 94 & -0.83 & 0.14 \\
\hline Current smokers & 48 & 433 & -0.74 & 0.41 \\
\hline Marital status & & & & \\
\hline Never married & 5 & 49 & 1.9 & 0.08 \\
\hline Not married currently & 6 & 55 & $-2 \cdot 0$ & 0.07 \\
\hline Married currently & 89 & 797 & Ref. & \\
\hline Education (years) & & & 0.51 & $<0.001$ \\
\hline Mean & & & & \\
\hline SE & & & & \\
\hline $\mathrm{BMI}\left(\mathrm{kg} / \mathrm{m}^{2}\right)$ & & & -0.01 & 0.87 \\
\hline Mean & & & & \\
\hline SE & & & & \\
\hline Systolic blood pressure $(\mathrm{mmHg})$ & & & 0.04 & 0.012 \\
\hline Mean & & & & \\
\hline SE & & & & \\
\hline Plasma total cholesterol (mmol/l) & & & 0.01 & 0.09 \\
\hline Mean & & & & \\
\hline SE & & & & \\
\hline Plasma LDL-cholesterol (mmol/l) & & & 0.002 & 0.80 \\
\hline Mean & & & & \\
\hline SE & & & & \\
\hline Plasma HDL-cholesterol (mmol/l) & & & 0.02 & 0.34 \\
\hline Mean & & & & \\
\hline SE & & & & \\
\hline Plasma TAG (mmol/l) & & & 0.0009 & 0.75 \\
\hline Mean & & & & \\
\hline SE & & & & \\
\hline Modified Framingham Risk Score (units) & & & 0.07 & 0.55 \\
\hline Mean & & & & \\
\hline SE & & & & \\
\hline Use of antihypertensives & 5 & 43 & $-1 \cdot 4$ & 0.26 \\
\hline
\end{tabular}

Ref., referent value.

* All $P$ values were corrected for clustering within a twin pair using linear mixed models for continuous variables. Raw values for continuous variables are presented. Diet scores were continuous dependent variables. A regression coefficient was explained as the expected change in a diet score for 1 -unit increment in an independent variable (continuous) or the exposure relative to non-exposure group. 


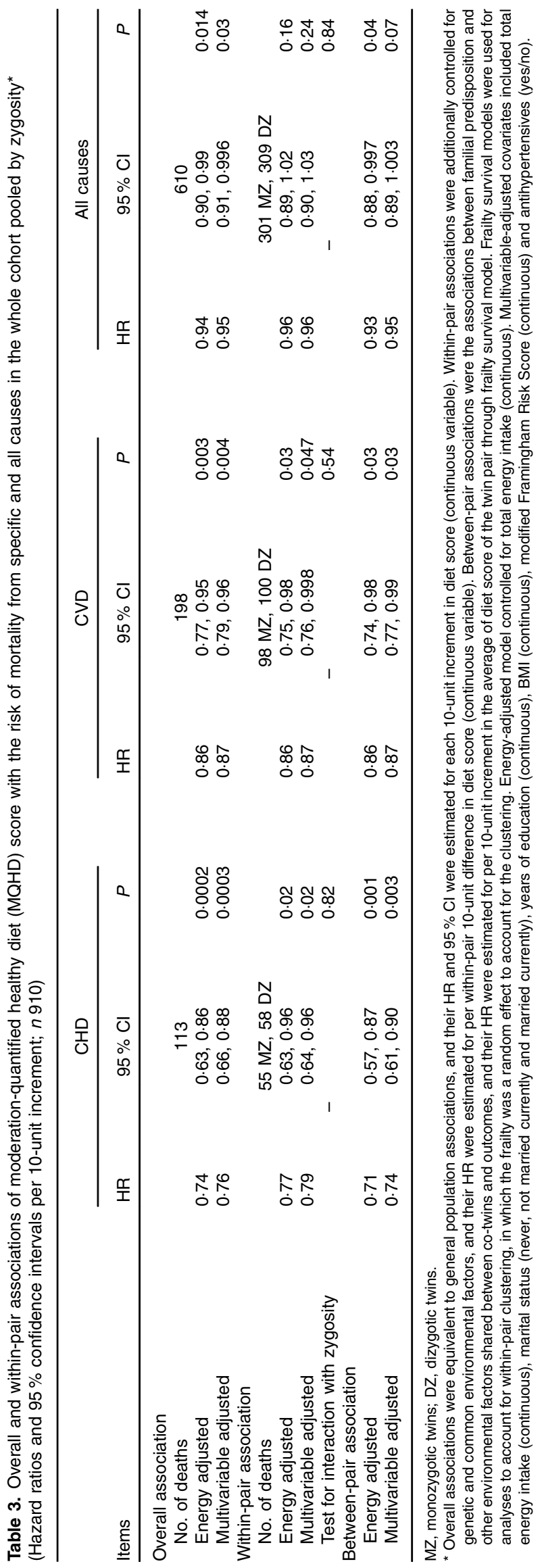

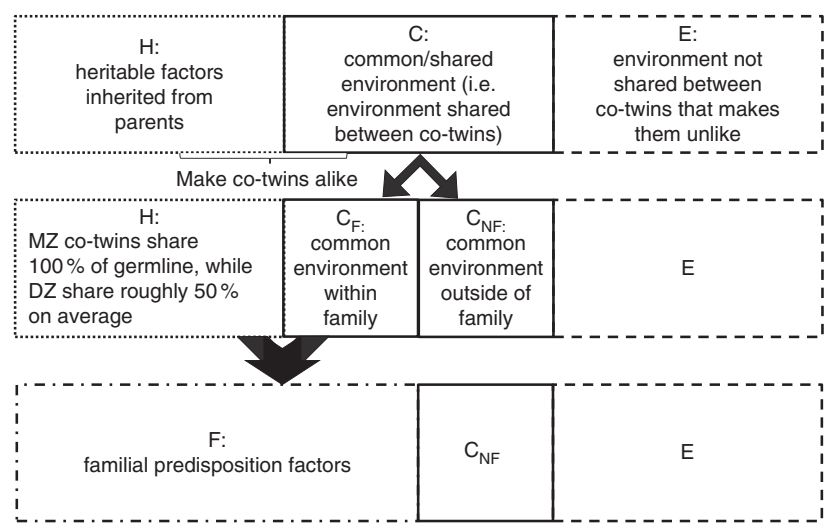

Fig. 1. Interpretation of factors shared between co-twins. MZ, monozygotic twins; DZ, dizygotic twins.

\section{Within-pair associations}

The interaction between within-pair effects and zygosity was not significant for specific or all causes (Table 3). In the whole cohort pooled by zygosity (i.e. MZ and DZ combined), for MQHD score, the within-pair association was statistically significant for specific causes after both energy and multivariable adjustment: multivariable-adjusted HR for a twin with a 10-unit higher MQHD score than his co-twin brother was 0.79 (95\% CI $0.64,0.96$ ) for CHD mortality risk and 0.87 (95\% CI $0 \cdot 76,0 \cdot 998)$ for cardiovascular mortality risk (Table 3 ).

\section{Between-pair associations}

Table 3 shows that the combined familial and shared nonfamilial factors (i.e. genetic and common environmental factors) significantly influenced the association between diet score and CHD death risk.

The confirmatory analysis demonstrated that multivariableadjusted HR for twins in the top quartile relative to the bottom quartile for MQHD score for CHD was statistically significant (Table 4), supporting our primary results. Similar results were obtained for aMQHD (online Supplementary Table S2).

We performed sensitivity analyses using several versions of slightly modified diet scores. Results were roughly similar after use of one weekly serving of red meat as the cutoff for the maximal score, exclusion of butter from dairy products, exclusion of both fat-rich potatoes (fried potatoes and potato/maize chips) from potato products and sweet grain products (donuts, cakes, pies and pastries) from grain products, separately (data not shown), and exclusion of alcohol intake as a diet score component (online Supplementary Table S3). The excluded food item(s) or groups were controlled for in the model. In another sensitivity analysis of twins after exclusion of those with baseline diabetes and CHD, similar results were obtained (data not shown).

We performed secondary analysis of intakes of nutrients, as shown in Table 5. As secondary analyses, the overall association was significant between Trichopoulou's Mediterranean diet score and the long-term risk of death from CHD and total CVD (online Supplementary Tables S4 and S5), but both within-pair 
Table 4. Overall associations of moderation-quantified healthy diet (MQHD) and alternative moderation-quantified healthy diet with coronary heart mortality risk comparing the top quartile with the bottom quartile*

(Hazard ratios (HR) and $95 \%$ confidence intervals)

\begin{tabular}{|c|c|c|c|c|c|c|}
\hline \multirow[b]{2}{*}{ Items } & \multicolumn{3}{|c|}{ MQHD score } & \multicolumn{3}{|c|}{ aMQHD score } \\
\hline & $\mathrm{HR}$ & $95 \% \mathrm{Cl}$ & $P$ & $\mathrm{HR}$ & $95 \% \mathrm{Cl}$ & $P$ \\
\hline No. of deaths & \multicolumn{2}{|c|}{53} & & \multicolumn{2}{|c|}{58} & \\
\hline Multivariable adjusted & 0.62 & $0.42,0.91$ & 0.014 & 0.68 & $0.48,0.96$ & 0.03 \\
\hline
\end{tabular}

aMQHD, alternative modified Mediterranean-style diet.

* HR was estimated through frailty survival model. Multivariable-adjusted covariates included total energy intake (continuous), marital status (never, not married currently and married currently), years of education (continuous), BMI (continuous), modified Framingham Risk Score (continuous) and antihypertensives (yes/no).

Table 5. Macronutrient intake among twins

(Mean values with their standard errors estimated from mixed model; $n 910$ )

\begin{tabular}{lrc}
\hline Macronutrients & Mean & SE \\
\hline Total fat $(\mathrm{g} / \mathrm{d})$ & 91 & 1.3 \\
Protein $(\mathrm{g} / \mathrm{d})$ & 75 & 0.8 \\
Carbohydrates $(\mathrm{g} / \mathrm{d})$ & 225 & 2.9 \\
SFA $(\mathrm{g} / \mathrm{d})$ & 36 & 0.5 \\
MUFA $(\mathrm{g} / \mathrm{d})$ & 48 & 0.7 \\
PUFA (g/d) & 8 & 0.1 \\
\hline
\end{tabular}

and between-pair effects were important in the overall associations (online Supplementary Table S4).

\section{Discussion}

A greater MQHD score was associated with a lower 40-year mortality risk from specific and all causes after adjustment for known CHD risk factors including socio-economic, lifestyle and clinical risk factors; however, after additional controlling for familial and shared non-familial factors, the association remained robust for $\mathrm{CHD}$ and cardiovascular mortality risk. In brief, the cardio-protective environmental factors that we demonstrated were predominantly nutritional.

\section{Interpretation of associations between whole diet and outcomes}

Our overall association was equivalent to that found in the general population. Different dietary patterns as previously reviewed $^{(12)}$, including Mediterranean $\operatorname{diet}^{(13,41)}$, alternative Mediterranean diet, Dietary Approaches to Stop Hypertension $\operatorname{diet}^{(14)}$ and animal protein diet ${ }^{(42)}$, had been linked to the risk of mortality from $\mathrm{CHD}^{(13-15,41,42)}, \mathrm{CVD}^{(14-16,41-43)}$ and all causes ${ }^{(13,41,43)}$. Our findings were generally consistent with previous studies in supporting the influence of a whole diet on specific and all-cause mortality risk; however, we provided long-term time-to-death evidence with the novel moderationquantified healthy dietary pattern.

More importantly, using the co-twin control-matched design, to our knowledge, we elucidated for the first time that the association between scales representative of a whole diet and the long-term mortality risk from $\mathrm{CHD}$ was free of potential confounding from familial factors. We additionally controlled for environmental factors shared between co-twins outside of family.

\section{Potential mechanisms underlying the association}

The underlying mechanism had been explored from various perspectives, including individual food components ${ }^{(44)}$, psychosocial health $^{(45,46)}$ and pathophysiological pathways such as inflammation ${ }^{(47,48)}$, oxidative stress $^{(49)}$, hypertension $^{(50)}$, autonomic ${ }^{(51)}$ and endothelial dysfunction ${ }^{(48,51)}$. It is well known that a diet rich in plant foods and fish is associated with a low risk for $\mathrm{CHD}$ and $\mathrm{CVD}^{(52)}$. However, our data supported the crucial influence of whole diet rather than just its individual components on the mortality risk.

\section{Limitations and strengths}

There are several limitations in our study. The NHLBI Twin Study only collected baseline dietary data. This would unlikely alter our findings, as a single measure would attenuate the association $^{(53)}$. Similar to other diet score constructions ${ }^{(13-16,47,50)}$, our cutoff points for scoring and the penalty assignment were arbitrary. Our findings supported that whole diet incorporating the quantified concept of moderate consumption was cardio-protective. Although alcohol is a component of a whole diet, the American Heart Association ${ }^{(54)}$ cautions people not to start drinking if they do not already drink alcohol. No baseline physical activity data were collected. Alternatively, we controlled for baseline BMI, an indicator for medium- to long-term nutritional status, and activity level reflecting dietary consumption relative to physical activity. The participants in our study were white male twins only. There should be caution in generalising our findings to females and other racial groups.

Our study has several advantages. By taking advantage of the accuracy of our dietary data, we were able to provide a quantitative reference for the moderate consumption in the practical implementation. In dietary planning and implementation, some of the energy content from solid fats and added sugars may be used for alcohol consumption instead ${ }^{(17)}$. Because each twin pair member shares the same birth date and co-twins are subject to the same age and period changes during follow-up, within-pair associations are controlled for age, cohort, period effects and secular trends as unmeasured (latent) environmental factors. 
In conclusion, the association of MQHD and aMQHD with a lower long-term CHD mortality risk is affected by nutritional and familial factors, supporting their use for dietary planning to prevent CHD mortality. Our findings support the current healthy diet concept, including moderate consumption of food items/groups, with an emphasis on whole diet.

\section{Acknowledgements}

Part of this work was performed when J. D. worked at Indiana University School of Public Health-Bloomington, and at the Division of Epidemiology, the Department of Medicine and Vanderbilt Center for Translational and Clinical Cardiovascular Research in Vanderbilt University Medical Center.

This study was supported by the American Heart Association (Scientist Development grant no. 10SDG2630182 to J. D.), and NHLBI grant (HL51429 to the NHLBI Twin Study).

J. D. designed the research, analysed the data and wrote the paper, and had primary responsibility for the final content of the manuscript. T. R. provided data from the NHLBI Twin Study and R. E. K. provided data on vital status and causes of death. All authors provided critical revisions to the manuscript for important intellectual contents; satisfied the authorship criteria of the International Committee of Medical Journal Editors; conducted research and provided essential materials; and read and approved the final manuscript.

None of the authors has any conflicts of interest to declare.

\section{Supplementary material}

For supplementary material/s referred to in this article, please visit http://dx.doi.org/10.1017/S0007114516001914

\section{References}

1. Go AS, Mozaffarian D, Roger VL, et al. (2014) Heart disease and stroke statistics - 2014 update: a report from the American Heart Association. Circulation 129, e28-e292.

2. Ergin A, Muntner P, Sherwin R, et al. (2004) Secular trends in cardiovascular disease mortality, incidence, and case fatality rates in adults in the United States. Am J Med 117, 219-227.

3. Kant AK \& Graubard BI (2006) Secular trends in patterns of self-reported food consumption of adult Americans: NHANES 1971-1975 to NHANES 1999-2002. Am J Clin Nutr 84, $1215-1223$.

4. Carmelli D, Selby JV, Quiroga J, et al. (1994) 16-year incidence of ischemic heart disease in the NHLBI twin study. a classification of subjects into high- and low-risk groups. Ann Epidemiol 4, 198-204.

5. Wu S-H, Neale MC, Acton AJ Jr, et al. (2014) Genetic and environmental influences on the prospective correlation between systemic inflammation and coronary heart disease death in male twins. Arterioscler Thromb Vasc Biol 34, 2168-2174.

6. Hasselbalch AL, Heitmann BL, Kyvik KO, et al. (2008) Studies of twins indicate that genetics influence dietary intake. $J$ Nutr 138, 2406-2412.

7. Pimpin L, Ambrosini GL, Llewellyn CH, et al. (2013) Dietary intake of young twins: nature or nurture? Am J Clin Nutr $\mathbf{9 8}$, 1326-1334.
8. Dubois L, Diasparra M, Bedard B, et al. (2013) Genetic and environmental influences on eating behaviors in 2.5-and 9-year-old children: a longitudinal twin study. Int J Behav Nutr Phys Act 10, 134.

9. Hammar N, Hakala P, Jorgensen L, et al. (2009) Migration and differences in dietary habits - a cross sectional study of Finnish twins in Sweden. Eur J Clin Nutr 63 , 312-322.

10. Hedlund E, Kaprio J, Lange A, et al. (2007) Migration and coronary heart disease: a study of Finnish twins living in Sweden and their co-twins residing in Finland. Scand J Public Health 35, 468-474.

11. Jacobs DR Jr \& Tapsell LC (2013) Conference on 'Translating nutrition: integrating research, practice and policy' Plenary Lecture II: food synergy: the key to a healthy diet. Proc Nutr Soc 72, 200-206.

12. Dai J (2008) Protective mechanisms of the Mediterranean diet on cardiovascular disease: a twin study. Doctoral Dissertation, Emory University, Atlanta, GA. Retrieved from ProQuest Dissertations and Theses (Accession Order No. AAT 3344491).

13. Trichopoulou A, Costacou T, Bamia C, et al. (2003) Adherence to a Mediterranean diet and survival in a Greek population. N Engl J Med 348, 2599-2608.

14. Salehi-Abargouei A, Maghsoudi Z, Shirani F, et al. (2013) Effects of Dietary Approaches to Stop Hypertension (DASH)style diet on fatal or nonfatal cardiovascular diseases incidence: a systematic review and meta-analysis on observational prospective studies. Nutrition 29, 611-618.

15. Struijk EA, May AM, Wezenbeek NL, et al. (2014) Adherence to dietary guidelines and cardiovascular disease risk in the EPIC-NL cohort. Int J Cardiol 176, 354-359.

16. Gardener H, Wright CB, Gu Y, et al. (2011) Mediterraneanstyle diet and risk of ischemic stroke, myocardial infarction, and vascular death: the Northern Manhattan Study. Am J Clin Nutr 94, 1458-1464.

17. U.S. Department of Agriculture \& U.S. Department of Health and Human Services (2010) Dietary Guidelines for Americans, 2010, 7th ed. Washington, DC: U.S. Government Printing Office.

18. Martinez-Gonzalez MA, Salas-Salvado $\mathrm{J}$, Estruch $\mathrm{R}$, et al. (2015) Benefits of the Mediterranean diet: insights from the PREDIMED study. Prog Cardiovasc Dis 58, 50-60.

19. Grosso G, Mistretta A, Frigiola A, et al. (2014) Mediterranean diet and cardiovascular risk factors: a systematic review. Crit Rev Food Sci Nutr 54, 593-610.

20. Sofi F, Macchi C, Abbate R, et al. (2014) Mediterranean diet and health status: an updated meta-analysis and a proposal for a literature-based adherence score. Public Health Nutr 17, 2769-2782.

21. Rumawas ME, Dwyer JT, McKeown NM, et al. (2009) The development of the Mediterranean-style dietary pattern score and its application to the American diet in the Framingham Offspring Cohort. J Nutr 139, 1150-1156.

22. Dai J, Krasnow R, Liu L, et al. (2013) The association between postload plasma glucose levels and 38-year mortality risk of coronary heart disease: the prospective NHLBI Twin Study. PLOS ONE 8, e69332.

23. Fabsitz RR, Kalousdian S, Carmelli D, et al. (1988) Characteristics of participants and nonparticipants in the NHLBI Twin Study. Acta Genet Med Gemellol (Roma) 37, 217-228.

24. Dai J, Mukamal KJ, Krasnow RE, et al. (2015) Higher usual alcohol consumption was associated with a lower 41-y mortality risk from coronary artery disease in men independent of genetic and common environmental factors: the prospective NHLBI Twin Study. Am J Clin Nutr 102, 31-39. 
25. Reed T, Carmelli D, Christian JC, et al. (1993) The NHLBI male veteran twin study data. Genet Epidemiol 10, 513-517.

26. Hauret KG, Bostick RM, Matthews CE, et al. (2004) Physical activity and reduced risk of incident sporadic colorectal adenomas: observational support for mechanisms involving energy balance and inflammation modulation. Am J Epidemiol 159, 983-992.

27. Feinleib M, Garrison RJ, Fabsitz R, et al. (1977) The NHLBI twin study of cardiovascular disease risk factors: methodology and summary of results. Am J Epidemiol 106, 284-285.

28. Mann GV, Pearson G, Gordon T, et al. (1962) Diet and cardiovascular disease in the Framingham study. i. measurement of dietary intake. Am J Clin Nutr 11, 200-225.

29. Dawber TR, Pearson G, Anderson P, et al. (1962) Dietary assessment in the epidemiologic study of coronary heart disease: the Framingham study. II. Reliability of measurement. Am J Clin Nutr 11, 226-234

30. Steffen LM, Van Horn L, Daviglus ML, et al. (2014) A modified Mediterranean diet score is associated with a lower risk of incident metabolic syndrome over 25 years among young adults: the CARDIA (Coronary Artery Risk Development in Young Adults) study. Br J Nutr 112, 1654-1661.

31. Dai J, Su Y-X, Bartell S, et al. (2009) Beneficial effects of designed dietary fatty acid compositions on lipids in triacylglycerol-rich lipoproteins among Chinese patients with type 2 diabetes mellitus. Metabolism 58, 510-518.

32. Mikulec KH, Holloway L, Krasnow RE, et al. (2004) Relationship of endogenous sex hormones to coronary heart disease: a twin study. J Clin Endocrinol Metab 89, 1240-1245.

33. Wilson PW, D'Agostino RB, Levy D, et al. (1998) Prediction of coronary heart disease using risk factor categories. Circulation 97, 1837-1847.

34. Falconer D (1960) Introduction to Quantitative Genetics. New York: Ronald Press.

35. Gharibvand L \& Liu L (2009) Paper 237-2009. Analysis of survival data with clustered events. In Proceedings of the SAS@ Global Forum 2009 Conference. Cary, NC: SAS Institute Inc.

36. Harrell F (2001) Regression Modeling Strategies: With Applications to Linear Models, Logistic Regression, and Survival Analysis. New York: Springer-Verlag New York, Inc.

37. Fang J, Austin PC \& Tu JV (2009) Paper 252-2009. Test for linearity between continuous confounder and binary outcome first, run a multivariate regression analysis second. In Proceedings of the SAS Global Forum 2009 Conference. Cary, NC: SAS Institute Inc.

38. Carlin JB, Gurrin LC, Sterne JA, et al. (2005) Regression models for twin studies: a critical review. Int J Epidemiol 34, 1089-1099.

39. Winkleby MA, Jatulis DE, Frank E, et al. (1992) Socioeconomic status and health: how education, income, and occupation contribute to risk factors for cardiovascular disease. $\mathrm{Am} \mathrm{J}$ Public Health 82, 816-820.

40. Freudenheim JL, Johnson NE \& Wardrop RL (1987) Misclassification of nutrient intake of individuals and groups using one-, two-, three-, and seven-day food records. Am I Epidemiol 126, 703-713.

41. Knoops KT, de Groot LC, Kromhout D, et al. (2004) Mediterranean diet, lifestyle factors, and 10-year mortality in elderly European men and women: the HALE project. JAMA 292, 1433-1439.

42. Chen Y, McClintock TR, Segers S, et al. (2013) Prospective investigation of major dietary patterns and risk of cardiovascular mortality in Bangladesh. Int I Cardiol 167, 1495-1501.

43. Tognon G, Lissner L, Saebye D, et al. (2014) The Mediterranean diet in relation to mortality and CVD: a Danish cohort study. Br J Nutr 111, 151-159.

44. Mente A, de Koning L, Shannon HS, et al. (2009) A systematic review of the evidence supporting a causal link between dietary factors and coronary heart disease. Arch Intern Med 169, 659-669.

45. Ferranti EP, Dunbar SB, Higgins M, et al. (2013) Psychosocial factors associated with diet quality in a working adult population. Res Nurs Health 36, 242-256.

46. Chocano-Bedoya PO, O'Reilly EJ, Lucas M, et al. (2013) Prospective study on long-term dietary patterns and incident depression in middle-aged and older women. Am J Clin Nutr 98, 813-820

47. Dai J, Miller AH, Bremner JD, et al. (2008) Adherence to the Mediterranean diet is inversely associated with circulating interleukin-6 among middle-aged men: a twin study. Circulation 117, 169-175.

48. Lopez-Garcia E, Schulze MB, Fung TT, et al. (2004) Major dietary patterns are related to plasma concentrations of markers of inflammation and endothelial dysfunction. Am J Clin Nutr 80, 1029-1035.

49. Dai J, Jones DP, Goldberg J, et al. (2008) Association between adherence to the Mediterranean diet and oxidative stress. Am J Clin Nutr 88, 1364-1370.

50. Weng LC, Steffen LM, Szklo M, et al. (2013) A diet pattern with more dairy and nuts, but less meat is related to lower risk of developing hypertension in middle-aged adults: the Atherosclerosis Risk in Communities (ARIC) study. Nutrients 5. 1719-1733.

51. Dai J, Lampert R, Wilson PW, et al. (2010) Mediterranean dietary pattern is associated with improved cardiac autonomic function among middle-aged men: a twin study. Circ Cardiovasc Qual Outcomes 3, 366-373.

52. McEvoy CT, Temple N \& Woodside JV (2012) Vegetarian diets, low-meat diets and health: a review. Public Health Nutr 15, 2287-2294.

53. Willett W (1998) Nutritional Epidemiology, 2nd ed. New York, NY: Oxford University Press.

54. American Heart Association (2015) Alcohol and heart health AHA recommendation. https://www.heart.org/HEARTORG/ GettingHealthy/NutritionCenter/HealthyEating/Alcohol-andHeart-Health_UCM_305173_Article.jsp (accessed February 2015). 